\title{
Knowledge and attitude of primary healthcare workers towards rational prescription of Artemisinin-based combination therapy in the treatment of uncomplicated malaria in Ondo State, southwestern Nigeria
}

\author{
Baba Danjuma Oyinaka ${ }^{1, A-B, D-F} \oplus$, Nura Muhammad Umar ${ }^{2, A-C, E-F} \oplus, N^{2}$ afiu Aminu ${ }^{3, A, C-F} \oplus$, \\ Calistus A Akinleye ${ }^{4, A, C-F \oplus}$, Tanko Nuhu ${ }^{5, A-F \oplus}$ \\ ${ }^{1}$ Department of Planning, Research and Statistics, State Ministry of Health, Lafia, Nasarawa State, Nigeria \\ 2 School of Pharmaceutical Sciences, Universiti Sains Malaysia, Penang, Malaysia \\ ${ }^{3}$ Department of Pharmaceutics and Pharmaceutical Microbiology, Faculty of Pharmaceutical Science, Usmanu \\ Danfodiyo University, Sokoto, Nigeria \\ ${ }^{4}$ Department of Community Medicine, Faculty of Clinical Sciences, Ladoke Akintola University of Science and \\ Technology, Ogbomoso, Nigeria \\ ${ }^{5}$ Department of Pharmaceutics and Pharmaceutical Microbiology, Faculty of Pharmaceutical Sciences, Usmanu \\ Danfodiyo University Sokoto, Nigeria \\ A - Research concept and design, B - Collection and/or assembly of data, C - Data analysis and interpretation, \\ $D$ - Writing the article, E - Critical revision of the article, F - Final approval of article
}

\begin{abstract}
Baba Danjuma Oyinaka, Nura Muhammad Umar, Nafiu Aminu, Calistus A Akinleye, Tanko Nuhu. Knowledge and Attitude of Primary Healthcare Workers towards Rational Prescription of Artemisinin-Based Combination Therapy in the Treatment of Uncomplicated Malaria in Ondo State Southwestern Nigeria. J Pre-Clin Clin Res. 2021; 5-14. doi: 10.26444/jpccr/131762
\end{abstract}

\begin{abstract}
Introduction and objective. For an effective and desired outcome of therapy to be achieved, the technical capacity of the healthcare worker is a key factor. The aim of the study is to assess knowledge and attitudes of primary healthcare (PHC) workers towards rational artemisinin-based combination therapy (ACT) prescribed in the treatment of uncomplicated malaria.

Materials and method. This is a descriptive cross-sectional survey in which a self-administered structured questionnaire was used. A combination of stratified and multistage sampling techniques where utilized in the selection of the healthcare workers (HCWs).

Results. Of the 422 respondents, 100 (23.7\%) were males and 322 (76.3\%) were females. Respondents with less than five years in practice accounted for $36 \%$, while $64 \%$ had six years and above of practice. Community health extension workers (CHEW) accounted for 54\%, while $46 \%$ were either community health officers (CHO), nurses, midwives, Bachelors of Science (BSc) in community health, or with other qualifications. A total of 390 (92.4\%) were aware of the availability of ACTs and used the malaria treatment protocols, whereas 28 (6.6\%) still used monotherapy for simple malaria treatment, and $2.7 \%$ did not use any form of test before initiating treatment. Co-administration of non-ACTs with ACTs accounted for $12 \%$, with $88 \%$ prescribing paracetamol with ACTs. $67 \%$ of the respondents required capacity building in rational ACT use.

Conclusion. The majority of the respondents had the requisite knowledge and skills coupled with positive attitudes in prescribing ACTs. Some respondents could not distinguish between an ACTs and a monotherapy. This may affect the exact application of the malaria treatment protocols, especially at the PHC level.
\end{abstract}

\section{Key words}

ACTs, prescription, healthcare workers, knowledge, Nigeria

\section{INTRODUCTION}

Malaria is one of the major public health problems in Nigeria and is endemic to the whole country. In 2018, it was reported that most malaria cases occurred in the World Health Organization (WHO) African region, amounting to 213 million (93\%), with nineteen countries in sub-Saharan Africa and India carrying almost $85 \%$ of the global malaria burden [1]. It further reported that six countries accounted

Address for correspondence: Tanko Nuhu, Usmanu Danfodiyo University Sokoto, Garba Nadama Road, 840212, Sokoto, Nigeria

E-mail: nuhu.tanko@yahoo.com

Received: 30.09.32020; accepted: 17.12.2020; first published: 18.01.2021 for more than half of all malaria cases worldwide: Nigeria (25\%), the Democratic Republic of the Congo (12\%), Uganda (5\%), and Côte d'Ivoire, Mozambique and Niger (4\% each) [1].

Nearly $85 \%$ of global malaria deaths in 2018 were concentrated in 20 countries in the WHO African region and India; Nigeria suffering the highest death rate with virtually $24 \%$ of all global malaria deaths. There were approximately 155 million malaria cases in the 11 high burden to high impact (HBHI) countries in 2018, in which Nigeria and the Democratic Republic of the Congo (DR Congo) accounted for 84 million (54\%) of the total number of cases [1].

Malaria is caused by Plasmodium falciparum (P. falciparum), and the mosquitoes Anopheles gambiae, 
Anopheles funestus, Anopheles arabiensis, and Anopheles moucheti are the major vectors that causes transmission all year round [2]. P. falciparum is the most prevalent malaria parasite in the WHO African region, accounting for $99.7 \%$ of estimated malaria cases, with $94 \%$ of all malaria deaths in 2018 [1]. Resistance of P. falciparum across Africa to common affordable antimalarial drugs like chloroquine and sulphadoxine-pyrimethamine was high that it hampered malaria control efforts in the region [2]. In 2001, due to the rapid widespread of chemo-resistance in malaria endemic countries, health professionals attached to the WHO recommended the implementation of Artemisinin-based Combination Therapy (ACT) as first line treatment for the better management of uncomplicated malaria in infected patients as a fundamental replacement of monotherapies [3]. ACT was recommended even in resource-poor settings where multidrug resistant (MDR) P. falciparum is a problem [2]. The WHO recommended that combination treatment rather than monotherapy should be used in areas where MDR to $P$. falciparum is problematic [4-6]. Combination drugs act at different sites or have different mechanisms of action. ACTs has been shown in many studies to have higher and sustainable parasitological cure rates over monotherapies, thereby reducing therapeutic failure rates, including $P$. falciparum resistant strains in sub-Sahara Africa malaria endemic countrie, compared to monotherapies [4]. Therefore this facilitates achieving better cure rates and are more likely to delay development of parasitic resistance [6]. Despite the numerous options in the ACTs, the options under consideration in Nigeria are Amodiaquine-Artesunate, or Artemether-Lumefantrine [6].

The inappropriate, ineffective and inefficient use of drugs at health facilities are commonplace in many developing countries [7], and the successful implementation of the ACT treatment policy essentially depends upon adequate training and support for healthcare (HC) providers to advance their knowledge and to ensure their acceptance and adherence to the new treatment guidelines [8]. However, correct knowledge of healthcare providers (HCPs) does not primarily translate into adherence to treatment guidelines [8]. For efficient and effective use of any medication in the treatment of a particular disease or medical problem, the attending HCW must have a great deal of knowledge of the disease, methods of diagnosing the disease, and how the patient will be monitored for effectiveness [9].

The rationale behind targeting primary healthcare (PHC) providers in this study is due to their seeming role as the first tier in the HC system where they are the first to come into contact with patients, thereby managing health related issues including malaria cases. It is very obvious that a great deal still needs to be done in ascertaining the capacities of HCWs in the treatment of malaria in the PHC facilities.

\section{OBJECTIVE}

This aim of the study was to assess the knowledge and attitudes of primary HCWs in government healthcare facilities (HFs) towards rational prescription of ACTs, and to evaluate the diagnostic criteria used prior to the initiation of ACTs in the treatment of uncomplicated malaria in Ondo State, southwest Nigeria.

\section{MATERIALS AND METHOD}

Study location. The study was conducted in Ondo State, southwest Nigeria, a State that was created in 1976 from the defunct Western State. It has a land area of 14,793 square kilometers with its administrative capital in Akure. The estimated population of the State in 2019 was 5,082,414 (based on national population commission (NPC) 2006 census with a State annual growth rate of 3.0\%). The State has 18 local government areas which are divided into three senatorial districts; Ondo-North, Ondo-South and OndoCentral. Agriculture constitutes the main occupation of the people of the State. Cocoa is the leading farm produce in Ondo State.

Study design. The study was a descriptive cross-sectional survey using a structured questionnaire. In order to troubleshoot the questionnaire on the grounds of quality, possible difficulties were detected during filling-in the questionnaires and estimated time required for their completion. The questionnaire was pre-tested in two PHC facilities in the Lafia Local Government Area of Nasarawa State, with 30 skilled HCWs as respondents. From the result of the pre-test, appropriate adjustments were made to the questionnaire, including questions that were not easily understood by the respondents and were rephrased for better comprehension.

Study population. The sample size for the study was calculated using Leslie Fisher's formulae:

$\mathrm{n}=\mathrm{z}^{2} \mathrm{pq} / \mathrm{d}^{2}$ where $\mathrm{n}=$ initial sample size, $\mathrm{z}=1.96$ confidence, $\mathrm{p}=$ prevalence of occurrence at $50 \%(0.5)$, $q=(1-p)=1-0.5=0.5, d=$ degree or margin of error 0.05

Using the above formula, the sample size was calculated as follows:

$$
\begin{gathered}
\mathrm{n}=1.96^{2 \star} 0.5^{\star} 0.5 \\
(0.05)^{2} \\
=384+10 \% \text { of } \mathrm{n} \\
\mathrm{n}=384+38=422
\end{gathered}
$$

The study population were nurses and Community Health Workers (CHWs), including officers-in-charge (OIC) of PHC facilities who are responsible for providing malaria treatment services to the community. All skilled HCWs in the selected public PHC facilities were included, while non-technical staff and unskilled HCWs were excluded.

Sampling technique. A combination of stratified and multistage sampling were utilized in the selection of the HCWs as respondents from the HFs. All HCWs were drawn from the various primary care HFs, first by LGA and then further disaggregated by HFs category. These categories were health post for category 1 , primary health clinic/basic health centre - category 2, and PHC centre/comprehensive health centre - category 3 .

The total number of skilled HCWs in each LGA were obtained from the department of planning, research and statistics, Ondo State Primary Healthcare Development Agency (OSPHCDA). A proportional allocation of the calculated sample size of skilled HCWs based on the 
categorization and number of HFs for each LGA were obtained. This was followed by a stratified sampling of the HCWs.

Research instruments. A semi-structured questionnaire designed to include: demography, open and close ended questions with some measure of diagnostic criteria were used as the quantitative data instrument which was administered on skilled HCWs providing malaria treatment services in the sampled HFs. The questionnaire consisted of four sections: socio-demographic data, knowledge about the subject, medicine sources to the HFs and the type of generic and other prescribed medicines often used, factors influencing the choice of product selected for use, and attitudes of the HCWs towards rational ACT prescribing.

Data collection. A quantitative data collection method was employed using structured self-administered questionnaires. A total of 422 questionnaire were administered and retrieved, sorted and the data were entered into a designed excel template, and finally cleaned before paving the way for data analysis.

Study variables. Dependent variables: knowledge acquired and attitudes exhibited in providing malaria treatments, while the independent variable were the socio-demographic characteristics and factors influencing choices of the therapeutic agents.

Statistical analysis. Data were checked for completeness and accuracy. All data from the field were compiled and entered in the statistical software tool (SPSS version 23). Frequencies, proportions, percentages, means for variables of interest were obtained, and compared for the skilled HCWs and across the LGAs, and appropriate tables and graphs generated. T-test was used to compare means of normally distributed continuous variables. The level of statistical significance was set at $5 \%$.

Ethical consideration. The study was approved by the Ondo State Health Research Ethics Committee (OSHREC) (Protocol NO. OSHREC/07/04/20/269). The main objective of the study was explained to participants after which they signed an informed consent form to indicate their willingness to participate. Participants were also informed that participation is voluntary, and anonymity guaranteed.

\section{RESULTS}

A total of 422 healthcare workers were eligible for and participated in the research and responded to the questionnaire. All the questionnaires administered were retrieved with female respondents accounting for 322 (76.3\%) and their male counterparts 100 (23.7\%). The Mean \pm Standard deviation of age of the participants was 36.7 years \pm 10.0 years. The age range $20-30$ years constituted $34.8 \%$ of the total number of respondents, followed closely by $31-40$ years (32.2\%), then $41-50$ years (24.9\%), and the least from 51 - 60 years (8.1\%). Socio-demographic characteristics of the HCWs is shown in Table 1.

The majority of the participants - 155 (36.3\%) had $<5$ years of work experience. This was followed by $6-10$ years
Table 1. Socio-demographic characteristics of the healthcare workers $(\mathrm{N}=422)$

\begin{tabular}{lcc}
\hline $\begin{array}{l}\text { Socio-demographic } \\
\text { characteristics of respondents }\end{array}$ & Frequency $(\mathrm{N})$ & Percentage (\%) \\
\hline Age: (Mean \pm SD) & $36.7 \pm 10.0$ & \\
$20-30$ & 147 & 34.8 \\
$31-40$ & 136 & 32.2 \\
$41-50$ & 105 & 24.9 \\
$51-60$ & 34 & 8.1 \\
Total & $\mathbf{4 2 2}$ & $\mathbf{1 0 0}$ \\
\hline Gender & & \\
Male & 100 & 23.7 \\
Female & 322 & 76.3 \\
\hline
\end{tabular}

(27.0\%) of work experience, then $11-15$ years (13.5\%). The least frequency was obtained from respondents with $>31$ years $(1.8 \%)$. The duration of practice and work experience is shown in Figure 1.

Educational qualifications of participants across the HCWs showed that community health extension workers (CHEW) accounted for 53.6\%, while nurses accounted for $16.4 \%$. Community health officers (CHO) and Bachelor of Science community health (BSc) accounted for $7.1 \%$ each, with midwives and other qualification representing $6.4 \%$ and $9.4 \%$, respectively.

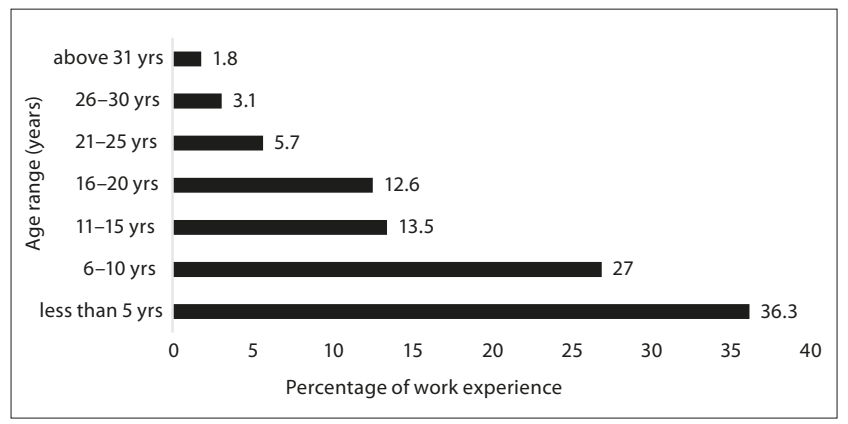

Figure 1. Duration of practice and work experience

Based on the level of PHC where respondents were working, health centres had the highest number of respondents (87.5\%), while the least number of respondents were from health post (1.4\%). Similarly, ranks of participants were widespread with OIC constituting 30 (7.1\%), assistant OIC accounted for 31 (9.5\%) and other ranks were 321 (76.1\%) (Tab. 2).

Awareness of the existence of a national malaria treatment protocol (NMTP) among the study participants revealed that $402(95.3 \%)$ were aware protocol, while 20 (4.7\%) were not aware. Of those who were aware of the existence of an NMTP, $390(92.4 \%)$ had a NMTP and made use of it, 21 (5.0\%) did not have but made use of it, while 11 (2.6\%) did not always make use of an NMTP. The reasons for non-reference to the protocol while prescribing ACTs revealed that 28 (87.5\%) did so for lack of understanding, and four (12.5\%) did so because they saw no need for its use (Tab. 2).

The majority of the participants chose ACTs as their preferred choice rather than monotherapy in the treatment of simple malaria, while Artemeter injection was the most preferred monotherapy. Among the ACTs, the most prescribed was Artemether-Lumefantrine 355 (88.1\%) (Tab. 2).

Rapid diagnostic test (RDT) 393 (95.4\%) was the preferred test before making diagnosis and the least was microscopy - eight (1.9\%). A very good number of the respondents 
Table 2. Knowledge and diagnostic criteria of respondents $(\mathrm{N}=422)$

\begin{tabular}{lcc}
\hline Categories & Frequencies & Percentages \\
\hline Level of PHC & & \\
Health post & 6 & 1.4 \\
Health clinic & 47 & 11.1 \\
Health centre & 369 & 87.5 \\
Total & 422 & 100 \\
\hline Rank & & \\
OIC & 30 & 7.1 \\
Assistant OIC & 40 & 9.5 \\
Programme Officers & 31 & 7.3 \\
Others & 321 & 76.1 \\
Total & 422 & 100 \\
\hline
\end{tabular}

\begin{tabular}{lcc}
\hline Awareness of the existence of national malaria & & \\
treatment protocols & 402 & 95.3 \\
Yes & 20 & 4.7 \\
No & 0 & 0.0 \\
Not sure & 422 & 100 \\
Total &
\end{tabular}

Availability and use of the protocol in health

$\begin{array}{lcc}\text { facility } & & \\ \text { Yes } & 390 & 92.4 \\ \text { No } & 21 & 5.0 \\ \text { Not always } & 11 & 2.6\end{array}$

\section{Reason for prescribing ACTs without referring to}

the protocols

\begin{tabular}{lcc} 
Lack of understanding & 28 & 87.5 \\
No need for its use & 4 & 12.5 \\
\hline
\end{tabular}

Drug(s) of choice in the treatment of simple malaria

Monotherapy

\begin{tabular}{cc}
394 & 93.4 \\
28 & 6.6 \\
\hline
\end{tabular}

Monotherapy used

Chloroquine

Sulphadoxine - Pyrimethamine

Artesunate

Amodiaquine

Artemether injection

Quinine

Preferred ACTs always prescribed

Artemether-Lumefantrine

Artesunate-Amodiaquine

Dihydroartemisinine-Piperaquine

None

\begin{tabular}{cc}
4 & 16.0 \\
2 & 8.0 \\
0 & 0 \\
0 & 0 \\
19 & 76.0 \\
0 & 0 \\
\hline & \\
355 & 88.1 \\
46 & 11.4 \\
0 & 0 \\
2 & 0.5 \\
\hline
\end{tabular}

(91.3\%) prescribed other drugs together with the ACTs, while only $1 \%$ did not prescribe other drugs with the ACTs. While prescribing other drugs with the ACTs, paracetamol accounted for $88.3 \%$ of these drugs prescribed. The number of drugs co-prescribed with ACTs shows that $85.2 \%$ of the respondents prescribed -2 drugs together with ACTs (Tab. 2).

The source of ACT supplies in the treatment of simple malaria assessed among the participants showed that 397 (94.1\%) community pharmacies were the points where most partic, and 19 (4.5\%) from central medical store. In checking stock status of ACT, the majority of respondents - 241 (68.5\%) stated that they checked manufacturing and expiry dates, while 105 (29.8\%) always checked for minimum stock (Tab. 2).

Generic ACTs 260 (61.8\%) were the most prescribed, while branded were the least prescribed, and the mixture of branded and generic accounted for 91 (21.6\%). The need for training on the rational use of ACTs was assessed and it was found that $292(69.5 \%)$ of the participants said they needed training on the rational use of ACT, while 95 (22.6\%) did not need training (Tab. 2).

\begin{tabular}{|c|c|c|}
\hline Categories & Frequencies & Percentages \\
\hline \multicolumn{3}{|c|}{ Type of test requested before making diagnosis } \\
\hline RDT & 393 & 95.4 \\
\hline Microscopy & 8 & 1.9 \\
\hline No test & 11 & 2.7 \\
\hline \multicolumn{3}{|l|}{ Prescribe drugs along with ACTs } \\
\hline Yes & 378 & 91.3 \\
\hline No & 4 & 1.0 \\
\hline Not always & 32 & 7.7 \\
\hline \multicolumn{3}{|c|}{ Medicines normally prescribed with ACTs } \\
\hline Vitamin C & 13 & 3.1 \\
\hline Vitamin B complex & 6 & 1.4 \\
\hline Multivitamins & 6 & 1.4 \\
\hline Paracetamol & 369 & 88.3 \\
\hline Antibiotics & 24 & 5.7 \\
\hline \multicolumn{3}{|c|}{ Number of drugs prescribed with ACTs } \\
\hline $1-2$ & 358 & 85.2 \\
\hline $3-4$ & 53 & 12.6 \\
\hline $5-6$ & 9 & 2.2 \\
\hline 7 and above & 0 & 0 \\
\hline \multicolumn{3}{|l|}{ Source of ACTs supplies } \\
\hline Community pharmacies & 397 & 94.1 \\
\hline Patent medicine & 6 & 1.4 \\
\hline Central Medical Store & 19 & 4.5 \\
\hline \multicolumn{3}{|l|}{ Always check stock status of ACTs } \\
\hline Yes & 344 & 82.1 \\
\hline No & 75 & 17.9 \\
\hline \multicolumn{3}{|l|}{ Stock status always check } \\
\hline Maximum stock & 6 & 1.7 \\
\hline Minimum stock & 105 & 29.8 \\
\hline Manufacturing and expiry date & 241 & 68.5 \\
\hline \multicolumn{3}{|l|}{ Presence of EML } \\
\hline Yes & 346 & 86.5 \\
\hline No & 54 & 13.5 \\
\hline \multicolumn{3}{|l|}{ Most ACTs prescribed } \\
\hline Generic & 260 & 61.8 \\
\hline Branded & 70 & 16.6 \\
\hline Mixture of branded and generic & 91 & 21.6 \\
\hline \multicolumn{3}{|c|}{ Need training on the rational use of ACTs } \\
\hline Yes & 292 & 69.5 \\
\hline No & 95 & 22.6 \\
\hline Not sure & 33 & 7.9 \\
\hline
\end{tabular}

$\mathrm{OIC}=$ Officer in-charge, EML = Essential medicine lists

The respondents' attitude toward malaria treatment were assessed using the Likert scale. On whether all malaria patients are tested before treatment with ACTs, 262 (62.1\%) strongly agreed while only $2(0.5 \%)$ strongly disagreed. Similarly, on the need to consult a malaria treatment guideline for all cases of malaria, 241 (57.1\%) strongly agreed on the importance to consult a malaria treatment guideline, while three (0.7\%) strongly disagreed (Tab. 3). A total of 226 (53.6\%l) strongly agreed that all malaria treatments should be handled by qualified health workers and 18 (4.3\%) strongly disagreed on malaria treatment being handled by qualified health workers. On procurement of all malaria drugs from an approved vendor, $150(35.6 \%)$ agreed, while $18(4.3 \%)$ strongly disagreed (Tab. 3).

The attitude towards prescribing other drugs with ACTs, showed that $283(67.1 \%)$ strongly disagreed that prescribing vitamins and minerals with ACTs can help stimulate a patient's appetite, while six (1.4\%) strongly agreed that prescribing vitamins and minerals with ACTs can help stimulate a patient's appetite. The attitude of respondents towards referrals when the patient shows danger signs 
Table 3. Assessment of attitudes of respondents using the Likert scale

\begin{tabular}{lcc}
\hline Description & Frequencies & Percentages \\
\hline All malaria patients are tested before treatment & & \\
with ACTs & & \\
$\quad$ Strongly agree & 262 & 62.1 \\
Agree & 139 & 32.9 \\
Undecided & 0 & 0 \\
Disagree & 19 & 4.5 \\
Strongly disagree & 2 & 0.5 \\
\hline
\end{tabular}

It is important to consult a malaria treatment

guideline for all cases of malaria

$\begin{array}{lcc}\text { Strongly agree } & 241 & 57.1 \\ \text { Agree } & 154 & 36.5 \\ \text { Undecided } & 6 & 1.4 \\ \text { Disagree } & 18 & 4.2 \\ \text { Strongly disagree } & 3 & 0.7\end{array}$

\begin{tabular}{lcc}
\hline $\begin{array}{l}\text { All malaria treatments are handled by qualified } \\
\text { health workers }\end{array}$ & \\
$\quad$ Strongly agree & 226 & 53.6 \\
Agree & 155 & 36.7 \\
Undecided & 30 & 7.1 \\
Disagree & 91 & 21.6 \\
$\quad$ Strongly disagree & 18 & 4.3 \\
\hline All malaria drugs are procured from an approved & & \\
vendor & & \\
Strongly agree & 133 & 31.5 \\
Agree & 150 & 35.6 \\
Undecided & 30 & 7.1 \\
Disagree & 91 & 21.6 \\
Strongly disagree & 18 & 4.3 \\
\hline
\end{tabular}

\begin{tabular}{lcc}
\hline Prescribing vitamins and minerals with ACTs can & & \\
help stimulate patient's appetite & 6 & 1.4 \\
Strongly agree & 23 & 5.5 \\
Agree & 0 & 0 \\
Undecided & 110 & 26.1 \\
Disagree & 283 & 67.1 \\
Strongly disagree &
\end{tabular}

\begin{tabular}{lcc}
\hline $\begin{array}{l}\text { Choose not to refer patient when the patient } \\
\text { shows danger signs }\end{array}$ & & \\
Strongly agree & 6 & 1.4 \\
Agree & 23 & 5.5 \\
Undecided & 0 & 0 \\
Disagree & 110 & 26.1 \\
Strongly disagree & 283 & 67.1 \\
\hline
\end{tabular}

revealed that 6(1.4\%) strongly agreed, while $283(67.1 \%)$ strongly disagreed (Tab. 3).

The bivariate analysis revealed an association between knowledge and attitude of the participants in prescribing treatment for simple malaria using the NMTP. It was found that the longer the duration of practice, the more positive the attitude of HCW in the rational treatment of simple malaria. Assessment of the association between all patients being tested before treatment and duration of practice was found to be significant: Fisher's exact test: 677.674, Df: 24; p-value $=0.000$ (Tab. 4).

Similarly, association between the importance to consult malaria guidelines in all cases of malaria and duration of practice was found to be significant with Fisher's exact test: 790.752, Df: 24; p-value $=0.000$. Association between all malaria treatment to be handled by qualified HCWs and duration of practice was found to be significant with Fisher's exact test: 646.338, Df: 24; p-value $=0.000$ (Tab. 4).

The association between drugs procured from approved vendors and duration of practice was found to be significant with Fisher's exact test: 683.870, Df: 24; p-value $=0.000$. Similarly, the association between prescribing vitamins and minerals with ACT and duration of practice was found to be significant with Fisher's exact test: 617.191, Df: 24; p-value = 0.000 . This implies that the longer the duration of practice, the more rational the attitude of the prescriber in prescription of simple malaria treatment. Association between the choice not to refer patient in the event patient showing danger signs and duration of practice was found to be significant with Fisher's exact test: 699.147, Df: 24; p-value $=0.000$ (Tab. 4).

The association between educational qualification of HCWs and their attitude in the treatment of simple malaria revealed that the more educated the HCWs, the more positive their attitude in the rational treatment of simple malaria. The association between all patients being tested before treatment and educational qualification was found to be significant with Fisher's exact test: 588.398, Df: 20; p-value $=0.000$ (Tab. 5).

Similarly, association between the need to consult malaria guidelines in all cases of malaria and educational qualification was found to be significant with Fisher's exact test test: 639.272 , Df: $20 ; \mathrm{p}$-value $=0.000$. Also the association between all malaria treatment handled by qualified HCWs and educational qualification was found to be significant with Fisher's exact test: 532.192, Df: 20; p-value $=0.000$. Likewise, the association between all malaria drugs procured from approved vendors and educational qualification was significant with Fisher's exact test: 646.787, Df: 20; $\mathrm{p}$-value $=0.000$. In the same manner, association between prescribing vitamins and minerals with ACT and educational qualification was significant with Fisher's exact test: 541.734, Df: 20; p-value $=0.000$ (Tab. 5).

Association between the HCW who may choose not to refer a patient, even when the patient showed danger signs, and educational qualification was found to be significant with Fisher's exact test: 786.115, Df: 20; p-value $=0.000$. This implies that more persons regardless of educational qualification disagreed with not referring a patient when the danger signs presents is the rational course of action (Tab. 5).

Table 6 shows the association between the ranks of HCWs and their attitude in the treatment of simple malaria. This is with aim to assess whether higher ranked HCWs act more rationally in the treatment of simple malaria than lower ranked HCWs. It was found that the higher the rank of HCWs, the more positive their attitude in the rational treatment of simple malaria. The association between all patients being tested before treatment and the rank of the consulting staff cutting across OIC, assistant OIC, programme officers and other staff, was significant with Fisher's exact test: 580.998, Df: $12 ;$ p-value $=0.000$.

Similarly, association between the need to consult malaria guidelines in all cases of malaria and rank was found to be significant with Fisher's exact test: 783.264, df: 12; p-value $=0.000$.

Association between all malaria treatments to be handled by qualified health workers and the rank of HCW was found to be significant with Fisher's exact test: 632.353, Df: 12; $\mathrm{p}$-value $=0.000$. Also, association between all malaria drugs procured from approved vendors and the rank of HCWs who prescribed and attended to patients who presented with simple malaria was found to be significant with Fisher's exact test: 626.070 , Df: 12 ; p-value $=0.000$. Similarly, association between prescribing vitamins and minerals with ACTs and rank of HCWs was significant with Fisher's exact test: 511.322, Df: 12 ; p-value $=0.000$. Association between the ability to choose not to refer patient, even when the patient showed 
Table 4. Assessment of association between duration of practice and attitude of healthcare workers in the treatment of simple malaria

\begin{tabular}{|c|c|c|c|c|c|c|c|c|}
\hline \multirow[b]{2}{*}{ Categories } & \multicolumn{8}{|c|}{ Duration of practice } \\
\hline & $<5$ yrs & $6-10 \mathrm{yrs}$ & $11-15 \mathrm{yrs}$ & $16-20 \mathrm{yrs}$ & $21-25 y r s$ & 26-30yrs & $>31 \mathrm{yrs}$ & Total \\
\hline \multicolumn{9}{|c|}{ All patients are tested before treatment } \\
\hline Strongly agree & $81(48.5)$ & $24(14.4)$ & $15(9.0)$ & $20(11.9)$ & $16(9.6)$ & $5(3.0)$ & $6(3.6)$ & 167 \\
\hline Agree & $46(39.7)$ & $37(31.9)$ & $20(17.2)$ & $8(6.9)$ & $2(1.7)$ & $3(2.6)$ & $0(0)$ & 116 \\
\hline Undecided & $3(10)$ & $4(13.3)$ & $5(16.7)$ & $12(40)$ & $0(0)$ & $4(13.3)$ & $2(6.7)$ & 30 \\
\hline Disagree & $22(24.2)$ & $45(49.5)$ & $15(16.5)$ & $9(9.9)$ & $0(0)$ & $0(0)$ & $0(0)$ & 91 \\
\hline Strongly disagree & $1(5.5)$ & $4(22.2)$ & $2(11.1)$ & $4(22.2)$ & $6(33.3)$ & $1(5.5)$ & $0(0)$ & 18 \\
\hline Total & $153(36.3)$ & $114(27.0)$ & $57(13.7)$ & $53(12.6)$ & $24(5.7)$ & $13(3.1)$ & $8(1.9)$ & 422 \\
\hline Fisher's exact test: 677.679 & Df: 24 & $P: 0.000$ & & & & & & \\
\hline \multicolumn{9}{|c|}{ It is important to consult malaria guideline for all cases of malaria } \\
\hline Strongly agree & $91(54.5)$ & $14(8.4)$ & $5(3.0)$ & $20(11.9)$ & $16(9.6)$ & $5(3.0)$ & $6(3.6)$ & 167 \\
\hline Agree & $36(31.0)$ & $47(40.5)$ & $30(25.9)$ & $8(6.9)$ & $2(1.7)$ & $3(2.6)$ & $0(0)$ & 116 \\
\hline Undecided & $3(10)$ & $4(13.3)$ & $5(16.7)$ & $12(40)$ & $0(0)$ & $4(13.3)$ & $2(6.7)$ & 30 \\
\hline Disagree & $22(24.2)$ & $45(49.5)$ & $15(16.5)$ & $9(9.9)$ & $0(0)$ & $0(0)$ & $0(0)$ & 91 \\
\hline Strongly disagree & $1(5.5)$ & $4(22.2)$ & $2(11.1)$ & $4(22.2)$ & $6(33.3)$ & $1(5.5)$ & $0(0)$ & 18 \\
\hline Total & $153(36.3)$ & $114(27.0)$ & $57(13.7)$ & $53(12.6)$ & $24(5.7)$ & $13(3.1)$ & $8(1.9)$ & 422 \\
\hline Fisher's exact test: 790.752 & Df: 24 & $\mathrm{P}: 0.000$ & & & & & & \\
\hline \multicolumn{9}{|c|}{ All malaria treatment is handled by qualified health workers } \\
\hline Strongly agree & $81(48.5)$ & $24(14.4)$ & $15(9.0)$ & $20(11.9)$ & $16(9.6)$ & $5(3.0)$ & $6(3.6)$ & 167 \\
\hline Agree & $46(43.4)$ & $37(4.9)$ & $10(9.4)$ & $8(7.6)$ & $2(1.9)$ & $3(2.8)$ & $0(0)$ & 106 \\
\hline Undecided & $3(10)$ & $4(13.3)$ & $5(16.7)$ & $12(40)$ & $0(0)$ & $4(13.3)$ & $2(6.7)$ & 30 \\
\hline Disagree & $22(24.2)$ & $45(49.5)$ & $15(16.5)$ & $9(9.9)$ & $0(0)$ & $0(0)$ & $0(0)$ & 91 \\
\hline Strongly disagree & $1(3.6)$ & $4(14.3)$ & $12(42.9)$ & $4(14.3)$ & $6(14.4)$ & $1(5.5)$ & $0(0)$ & 28 \\
\hline Total & $153(36.3)$ & $114(27.0)$ & $57(13.7)$ & $53(12.6)$ & $24(5.7)$ & $13(3.1)$ & $8(1.9)$ & 422 \\
\hline Fisher's exact test: 646.338 & Df: 24 & $\mathrm{P}: 0.000$ & & & & & & \\
\hline \multicolumn{9}{|c|}{ All malaria drugs are procured from approved vendors } \\
\hline$\underline{\text { Strongly agree }}$ & $3(50)$ & $2(33.3)$ & $1(16.7)$ & $0(0)$ & $0(0)$ & $0(0)$ & $0(0)$ & 6 \\
\hline Agree & $6(28.1)$ & $3(13.0)$ & $1(4.3)$ & $8(34.8)$ & $2(8.7)$ & $3(13.0)$ & $0(0)$ & 23 \\
\hline Undecided & $0(00)$ & $0(0)$ & $0(0)$ & $0(0)$ & $0(0)$ & $0(0)$ & $0(0)$ & 0 \\
\hline Disagree & $56(50.9)$ & $30(27.3)$ & $15(13.6)$ & $9(8.2)$ & $0(0)$ & $0(0)$ & $0(0)$ & 110 \\
\hline Strongly disagree & $120(42.4)$ & $65(23.0)$ & $40(14.1)$ & $25(8.8)$ & $15(5.3)$ & $15(5.5)$ & $3(1.1)$ & 283 \\
\hline Total & $185(43.8)$ & $100(23.7)$ & $57(13.5)$ & $42(10.0)$ & $17(4.0)$ & $18(4.3)$ & $3(0.7)$ & 422 \\
\hline Fisher's exact test: 683.870 & Df: 24 & P:0.000 & & & & & & \\
\hline \multicolumn{9}{|c|}{ Prescribing vitamins and minerals with ACT help stimulate patients appetite } \\
\hline Strongly agree & $91(54.5)$ & $14(8.4)$ & $5(3.0)$ & $20(11.9)$ & $16(9.6)$ & $5(3.0)$ & $6(3.6)$ & 167 \\
\hline Agree & $36(31.0)$ & $47(40.5)$ & $30(25.9)$ & $8(6.9)$ & $2(1.7)$ & $3(2.6)$ & $0(0)$ & 116 \\
\hline Undecided & $3(10)$ & $4(13.3)$ & $5(16.7)$ & $12(40)$ & $0(0)$ & $4(13.3)$ & $2(6.7)$ & 30 \\
\hline Disagree & $22(24.2)$ & $45(49.5)$ & $15(16.5)$ & $9(9.9)$ & $0(0)$ & $0(0)$ & $0(0)$ & 91 \\
\hline Strongly disagree & $1(5.5)$ & $4(22.2)$ & $2(11.1)$ & $4(22.2)$ & $6(33.3)$ & $1(5.5)$ & $0(0)$ & 18 \\
\hline Total & $153(36.3)$ & $114(27.0)$ & $57(13.7)$ & $53(12.6)$ & $24(5.7)$ & $13(3.1)$ & $8(1.9)$ & 422 \\
\hline Fisher's exact trest: 617.191 & Df: 24 & $P: 0.000$ & & & & & & \\
\hline \multicolumn{9}{|c|}{ Choose not to refer patient even when the patient showed danger signs } \\
\hline Strongly agree & $3(50)$ & $2(33.3)$ & $1(16.7)$ & $0(0)$ & $0(0)$ & $0(0)$ & $0(0)$ & 6 \\
\hline Agree & $6(28.1)$ & $3(13.0)$ & $1(4.3)$ & $8(34.8)$ & $2(8.7)$ & $3(13.0)$ & $0(0)$ & 23 \\
\hline Undecided & $0(0)$ & $0(0)$ & $0(0)$ & $0(0)$ & $0(0)$ & $0(0)$ & $0(0)$ & 0 \\
\hline Disagree & $56(50.9)$ & $30(27.3)$ & $15(13.6)$ & $9(8.2)$ & $0(0)$ & $0(0)$ & $0(0)$ & 110 \\
\hline Strongly disagree & $120(42.4)$ & $65(23.0)$ & $40(14.1)$ & $25(8.8)$ & $15(5.3)$ & $15(5.5)$ & $3(1.1)$ & 283 \\
\hline Total & $185(43.8)$ & $100(23.7)$ & $57(13.5)$ & $42(10.0)$ & $17(4.0)$ & $18(4.3)$ & $3(0.7)$ & 422 \\
\hline Fisher's exact test: 699.147 & Df: 24 & $\mathrm{P}: 0.000$ & & & & & & \\
\hline
\end{tabular}


Table 5. Assessment of association between educational qualification and attitude of health workers in the treatment of simple malaria

\begin{tabular}{|c|c|c|c|c|c|c|c|}
\hline \multirow[b]{2}{*}{ Categories } & \multicolumn{7}{|c|}{ Educational qualification } \\
\hline & CHEW & $\mathrm{CHO}$ & Nurse & Midwife & BSc C. Health & Others & Total \\
\hline \multicolumn{8}{|c|}{ All patients are tested before treatment } \\
\hline Strongly agree & $115(43.9)$ & $20(7.6)$ & $44(16.8)$ & $20(7.6)$ & $26(9.9)$ & $37(14.1)$ & 262 \\
\hline Agree & $102(73.4)$ & $5(3.6)$ & $20(14.4)$ & $5(3.6)$ & $4(2.9)$ & $3(2.6)$ & 139 \\
\hline Undecided & $0(10)$ & $0(13.3)$ & $0(16.7)$ & $0(40)$ & $0(0)$ & $0(13.3)$ & 0 \\
\hline Strongly disagree & $1(50.0)$ & $0(0)$ & $0(0)$ & $1(50.0)$ & $0(33.3)$ & $0(0)$ & 2 \\
\hline Total & $226(53.6)$ & $30(7.1)$ & $69(16.4)$ & $27(6.4)$ & $30(7.1)$ & $40(9.5)$ & 422 \\
\hline Fisher's exact test: 588.398 & Df: 20 & $P: 0.000$ & & & & & \\
\hline \multicolumn{8}{|c|}{ It is important to consult malaria guideline for all cases of malaria } \\
\hline Strongly agree & $111(45.6)$ & $20(8.2)$ & $40(16.5)$ & $15(16.2)$ & $20(9.9)$ & $37(8.2)$ & 243 \\
\hline Undecided & $0(10)$ & $0(13.3)$ & $0(16.7)$ & $0(40)$ & $0(0)$ & $0(13.3)$ & 0 \\
\hline Disagree & $8(42.1)$ & $5(26.3)$ & $5(26.3)$ & $1(5.3)$ & $0(0)$ & $0(0)$ & 19 \\
\hline Strongly disagree & $1(50.0)$ & $0(0)$ & $0(0)$ & $1(50.0)$ & $0(33.3)$ & $0(0)$ & 2 \\
\hline Total & $226(53.6)$ & $30(7.1)$ & $69(16.4)$ & $27(6.4)$ & $30(7.1)$ & $40(9.5)$ & 422 \\
\hline Fisher's exact test: 639.272 & Df: 20 & P:0.000 & & & & & \\
\hline \multicolumn{8}{|c|}{ All malaria treatment are handled by qualified health workers } \\
\hline Strongly agree & $111(72.5)$ & $7(4.6)$ & $12(7.8)$ & $0(0)$ & $14(9.2)$ & $9(5.9)$ & 153 \\
\hline Agree & $69(46.9)$ & $5(3.4)$ & $35(23.8)$ & $2(1.4)$ & $10(6.8)$ & $26(17.7)$ & 147 \\
\hline Undecided & $3(12.5)$ & $4(16.7)$ & $5(20.8)$ & $12(50)$ & $0(0)$ & $4(16.7)$ & 24 \\
\hline Disagree & $42(55.3)$ & $10(13.2)$ & $15(19.7)$ & $9(11.8)$ & $0(0)$ & $0(0)$ & 76 \\
\hline Strongly disagree & $1(5.6)$ & $4(22.2)$ & $2(11.1)$ & $4(22.2)$ & $6(33.3)$ & $1(5.6)$ & 18 \\
\hline Total & $226(53.6)$ & $30(7.1)$ & $69(16.4)$ & $27(6.4)$ & $30(7.1)$ & $40(9.5)$ & 422 \\
\hline \multicolumn{8}{|c|}{ All malaria drugs are procured from approved vendors } \\
\hline Strongly agree & $105(43.4)$ & $20(8.3)$ & $34(14.1)$ & $20(8.3)$ & $26(10.7)$ & $37(15.3)$ & 242 \\
\hline Agree & $112(70.4)$ & $5(3.1)$ & $30(18.9)$ & $5(3.1)$ & $4(2.5)$ & $3(1.9)$ & 159 \\
\hline Undecided & $0(10)$ & $0(13.3)$ & $0(16.7)$ & $0(40)$ & $0(0)$ & $0(13.3)$ & 0 \\
\hline Disagree & $8(42.1)$ & $5(26.3)$ & $5(26.3)$ & $1(5.3)$ & $0(0)$ & $0(0)$ & 19 \\
\hline Strongly disagree & $1(50.0)$ & $0(0)$ & $0(0)$ & $1(50.0)$ & $0(33.3)$ & $0(0)$ & 2 \\
\hline Total & $226(53.6)$ & $30(7.1)$ & $69(16.4)$ & $27(6.4)$ & $30(7.1)$ & $40(9.5)$ & 422 \\
\hline Fisher's exact test: 646.787 & Df: 20 & P:0.000 & & & & & \\
\hline
\end{tabular}

$\mathrm{CHEW}=$ Community Health Extension Worker, $\mathrm{CHO}=$ Community Health Officer, $\mathrm{BSc} \mathrm{C} .=$ Bachelor of Science Community Health

danger signs, and rank was found to be significant with Fisher's exact test: 564.900, Df: 12; p-value $=0.000$. (Tab. 6).

\section{DISCUSSION}

The attitude and level of knowledge of PHC workers towards the rational prescription of ACTs in the treatment of uncomplicated malaria in Ondo State Southwest Nigeria was investigated.

Healthcare providers working in PHC facilities anywhere in the world, including Nigeria, play a very vital role in delivering health care for patients with health-related challenges. This is because the PHCs are the first point of call for most people. The study established that the majority of the respondents were females, with most having less than five years experiences as health care providers. The preponderance of females may be linked to the notion that most health care givers at the PHC levels are nurses, midwives, and others also being mostly female.

The minimal qualification in the current study was that of a CHEW who represented the highest number of workers in the PHCs, the highest qualification was Bachelor of Science, and community health worker with the lowest qualification and number of respondents. The highest number of CHEWs in this study was in line with a related study where they reported CHEWs to be the highest [10]. The implication is that, despite having the requisite qualifications, and with little experience, the CHEWs might not be able to deliver on this mandate due to the strong association between knowledge and duration of practice. This also indicates that as one gains more experience in practice, there are high tendencies or chances of strict adherence to the service protocol due to regular training and re-training. However, malaria treatment guidelines have changed since the introduction of ACT in 2004. As a result, this was followed by a number of measures 
Table 6. Assessment of association between ranks of healthcare workers and their attitude towards the rational use of NMTP

\begin{tabular}{|c|c|c|c|c|c|}
\hline Categories & $\mathrm{OIC}$ & Asst. OIC & Program Officer & Others & Total \\
\hline \multicolumn{6}{|c|}{ All patients are tested before treatment } \\
\hline Strongly agree & $16(6.1)$ & $20(7.6)$ & $14(5.3)$ & $212(80.9)$ & 262 \\
\hline Agree & $12(8.6)$ & $15(10.8)$ & $12(8.6)$ & $100(71.9)$ & 139 \\
\hline Undecided & $0(0)$ & $0(0)$ & $0(0)$ & $0(0)$ & 0 \\
\hline Disagree & $1(42.1)$ & $5(26.3)$ & $5(26.3)$ & $8(42.1)$ & 19 \\
\hline Strongly disagree & $1(50.0)$ & $0(0)$ & $0(0)$ & $1(50.0)$ & 2 \\
\hline Total & $30(7.1)$ & $40(9.5)$ & $31(7.3)$ & $321(76.1)$ & 422 \\
\hline Fisher's exact test: 580.998 & Df: 12 & P:0.000 & & & \\
\hline \multicolumn{6}{|c|}{ It is important to consult malaria guideline for all cases of malaria } \\
\hline Strongly agree & $18(7.4)$ & $28(11.5)$ & $6(2.5)$ & $191(78.6)$ & 243 \\
\hline Agree & $10(6.3)$ & $7(4.4)$ & $20(12.7)$ & $121(76.6)$ & 158 \\
\hline Undecided & $0(0)$ & $0(0)$ & $0(0)$ & $0(0)$ & 0 \\
\hline Disagree & $1(5.3)$ & $5(26.3)$ & $5(26.3)$ & $8(42.1)$ & 19 \\
\hline Strongly disagree & $1(50.0)$ & $0(0)$ & $0(0)$ & $1(50.0)$ & 2 \\
\hline Total & $30(7.1)$ & $40(9.5)$ & $31(7.3)$ & $321(76.1)$ & 422 \\
\hline Fisher's exact test: 783.264 & Df: 12 & P:0.000 & & & \\
\hline \multicolumn{6}{|c|}{ All malaria treatment is handled by qualified health workers } \\
\hline Strongly agree & $5(3.9)$ & $5(3.9)$ & $4(3.1)$ & $104(81.3)$ & 128 \\
\hline Agree & $9(5.8)$ & $15(9.7)$ & $5(3.2)$ & $126(81.3)$ & 155 \\
\hline Undecided & $3(10.0)$ & $4(13.3)$ & $5(16.7)$ & $18(60)$ & 30 \\
\hline Disagree & $12(13.2)$ & $10(11)$ & $15(16.5)$ & $54(59.3)$ & 91 \\
\hline Strongly disagree & $1(5.6)$ & $6(33.3)$ & $2(11.1)$ & $9(50.0)$ & 18 \\
\hline Total & $30(7.1)$ & $40(9.5)$ & $31(7.3)$ & $321(76.1)$ & 422 \\
\hline Fisher's exact test: 632.353 & Df: 12 & P:0.000 & & & \\
\hline \multicolumn{6}{|c|}{ All malaria drugs are procured from approved vendors } \\
\hline Strongly agree & $5(3.7)$ & $5(3.7)$ & $4(3.0)$ & $119(89.5)$ & 133 \\
\hline Agree & $9(6)$ & $15(10)$ & $5(3.3)$ & $121(80.7)$ & 150 \\
\hline Undecided & $3(10.0)$ & $4(13.3)$ & $5(16.7)$ & $18(60)$ & 30 \\
\hline Disagree & $12(13.2)$ & $10(11)$ & $15(16.5)$ & $54(59.3)$ & 91 \\
\hline Strongly disagree & $1(5.6)$ & $6(33.3)$ & $2(11.1)$ & $9(50.0)$ & 18 \\
\hline Total & $30(7.1)$ & $40(9.5)$ & $31(7.3)$ & $321(76.1)$ & 422 \\
\hline Fisher's exact test: 626.070 & Df: 12 & P:0.000 & & & \\
\hline \multicolumn{6}{|c|}{ Prescribing vitamins and minerals with ACT help stimulate patients } \\
\hline Strongly agree & $1(16.7)$ & $2(33.3)$ & $2(33.3)$ & $1(16.7)$ & 6 \\
\hline Agree & $2(8.7)$ & $3(13.0)$ & $8(34.8)$ & $10(43.4)$ & 23 \\
\hline Undecided & $0(0)$ & $0(0)$ & $0(0)$ & $0(0)$ & 0 \\
\hline Disagree & $15(13.6)$ & $5(4.5)$ & $12(10.9)$ & $78(70.9)$ & 110 \\
\hline Strongly disagree & $12(4.2)$ & $30(10.6)$ & $9(3.2)$ & $232(82.0)$ & 283 \\
\hline Total & $30(7.1)$ & $40(9.5)$ & $31(7.3)$ & $321(76.1)$ & 422 \\
\hline Fisher's exact test: 511.322 & Df: 12 & $\mathrm{P}: 0.000$ & & & \\
\hline \multicolumn{6}{|c|}{ Choose not to refer patient even when the patient showed danger signs } \\
\hline Strongly agree & $1(16.7)$ & $2(33.3)$ & $2(33.3)$ & $1(16.7)$ & 6 \\
\hline Agree & $2(8.7)$ & $3(13.0)$ & $8(34.8)$ & $10(43.4)$ & 23 \\
\hline Undecided & $0(0)$ & $0(0)$ & $0(0)$ & $0(0)$ & 0 \\
\hline Disagree & $15(13.6)$ & $5(4.5)$ & 12(10.9) & $78(70.9)$ & 110 \\
\hline Strongly disagree & $12(4.2)$ & $30(10.6)$ & $9(3.2)$ & $232(82.0)$ & 283 \\
\hline Total & $30(7.1)$ & $40(9.5)$ & $31(7.3)$ & $321(76.1)$ & 422 \\
\hline Fisher's exact test: 564.900 & Df: 12 & $\mathrm{P}: 0.000$ & & & \\
\hline
\end{tabular}

OIC $=$ Officer in-charge 
to ensure its effective implementation, which include but were not limited to in-service training of health care providers to regularly update their knowledge and improve their skills [8].

On the awareness of NMTP, the majority were aware of its existence, and possession of the NMTP and ACTs is the preferred treatment option in the management of uncomplicated malaria. This is in tandem with studies by Kuete et al., who reported that health practitioners in HFs used the most commonly recommended ACTs for mild malaria treatment, therefore respecting the national and WHO guidelines on uncomplicated malaria management [4].

Diagnostic testing for malaria is the basis of the WHO initiative-T3 [10]. Therefore, the need to carry out investigations before initiating therapy can never be overemphasized. The WHO recommends that all persons of all ages in all epidemiological settings with suspected malaria should take a parasitological confirmation of diagnosis by either microscopy or RDT [10]. On the diagnostic criteria from this study, it was generally accepted that investigations using RDT were always used before initiation of ACT in uncomplicated malaria. A high utilization of RDT was also reported in a similar study in Lagos, southwest Nigeria [10], although this is in contrast to a similar study in which the physicians were reportedly not requesting RDT before making diagnosis [11]. Hence, there may be a high tendency of irrational medication which may not be cost effective for the patients. The use of RDT before initiating treatment will reduce the overburden of antimalarial drugs; therefore ensuring that treatment is only carried out on confirmed malaria cases and thereby preventing antimalarial drug resistance.

In addition to the afore-mentioned, the findings of the presented study revealed a low level of the use of microscopy in diagnosis. This was also reported by Asibong et al. who indicated that HCWs perceived RDT to be more effective for malaria diagnosis than microscopy and clinical diagnosis [10]. The results of this study also indicated that the various categories of HCWs at the PHC level showed that there existed a gap in the knowledge and attitude about the usage of RDT for diagnosis. Even though the majority of HCWs had the requisite qualification to assess patients and make such request to the laboratory, a few of them felt it was not necessary to carry out the RDT before making diagnosis. This, therefore, contradicts the WHO T3 policy of testing before treatment in all malaria cases, as contained in the NMTP [12].

The concomitant medications alongside ACTs may be explained by either the desire of the prescriber to rapidly clear most symptoms experienced by the patients, or sometimes by the insistence of patients to receive these drugs to improve their health [4]. The current findings revealed some salient issues regarding the majority of HCWs co-prescribing ACTs with at least another but non-antimalarial medication: such as vitamins and minerals, antibiotics and analgesics to control pyrexia, although the responsibility of managing fever cases, particularly malaria fever at the PHC level, depends on the skill of the HCWs. The most commonly prescribed concomitant medications in the presented study were analgesics. Co-prescription with vitamins, minerals and antibiotics was also practiced by the HCWs, but should be applied with caution because the use of ACTs with an antioxidant could be counter-productive due to the reaction of ACTs with anti-oxidants. Thorough assessment of the need must be carried out before administration, and must be spaced to avoid such interactions.

Another interesting finding in the current study is that most HCWs sourced their ACTs from approved and licensed pharmacies, while some never believed in procuring ACTs from a reputable source. This is because they prefer ACTs to be sourced form patent medicine vendors (PMVs). With this, the lives of suspecting malaria patients could be exposed to unwholesome products as most PMVs are controlled and owned by non-health care workers who know almost nothing beyond their trade, and also lack the requisite knowledge on the current NMTP. This will directly or indirectly affect the quality of care being provided. The current findings also showed that there is a strong association between the knowledge and attitude of HCWs about the source of the ACTs they stocked for dispensing in their pharmacies, which could ultimately lead to irrational prescribing of ACTs and may lead to treatment failure, and other adverse effects.

Nevertheless, in the management of malaria, the recommended medicines are to be used after proper patient assessment, and its subsequent classification of either being severe, uncomplicated or malaria in pregnancy, are readily made before treatment is initiated $[3,11,13]$. The medicines concerned are the ACTs which are recommended by the WHO as first line medicines for the treatment of uncomplicated malaria. It is expected that the proper and efficient utilization of the right inputs should generate the desired results.

Generally, the HCWs have positive attitudes towards rational ACT prescription in the management of uncomplicated malaria. It was also generally indicated that the level of knowledge of HCWs have a direct implication in their rational thinking and making the appropriate choices of the right therapeutic options. However, a sizeable number of participants would require further training in order to use the right protocols. This was seen in the area where some health workers would not like to carry out thorough investigations, as required before making an intervention.

\section{CONCLUSIONS}

The findings showed that, despite the positive attitudes demonstrated by HCWs in prescribing ACTs, a significant number could not distinguish between an ACT and a monotherapy and may find this difficult in using the standard treatment protocols. Also, from the number of medicines co-administered with the ACTs, the interactions with such drugs may be responsible for the lack of proper absorptions of the ACTs which lead to its ineffectiveness.

\section{REFERENCES}

1. World Health Organization. World Malaria Report 2019. Geneva. WHO Regional Office for Africa. 2019. 1-232 p.

2. Dawaki S, Al-Mekhlafi HM, Ithoi I, Ibrahim J, Atroosh WM, Abdulsalam AM, et al. Is Nigeria Winning The Battle Against Malaria? Prevalence, Risk Factors and KAP Assessment among Hausa Communities in Kano State. Malar J. 2016; 15(1): 1-14.

3. World Health Organization. Antimalarial Drug Combination Therapy: Report of a WHO Technical Consultation. World Heal Organ Geneva. $2001 ; 36$.

4. Kuete T, Essono Mvoa E, Yinyang J, Epanya Priso A, Gougue Chamabe PC, Mpondo Mpondo E, et al. Pattern of Uncomplicated Malaria Treatment and Antimalarial Prescription Practices among Health 
Workers in the Littoral Region of Cameroon: An Assessment of Ten Years Post-Malaria Treatment Policy Change. Pharmacol Pharm Pharm. 2016; 07(06): 217-225.

5. Gbotosho GO, Sowunmi A, Happi CT, Okuboyejo TM. Therapeutic Efficacies of Artemisinin-based Combination Therapies in Nigerian Children with Uncomplicated Falciparum Malaria during Five Years of Adoption as First-line Treatments. Am J Trop Med Hyg. 2011; 84(6): 936-943.

6. Meremikwu M, Alaribe A, Ejemot R, Oyo-Ita A, Ekenjoku J, Nwachukwu $\mathrm{C}$, et al. Artemether-lumefantrine versus Artesunate plus Amodiaquine for Treating Uncomplicated Childhood Malaria in Nigeria: Randomized Controlled Trial. Malar J. 2006; 5: 1-6.

7.Mannan AA, Malik EM, Ali KM. Antimalarial Prescribing and Dispensing Practices in Health Centres Of Khartoum State, 2003-04. East Mediterr Heal J. 2009; 15(1): 122-128.

8. Mannan AA, Elmardi KA, Idris YA, Spector JM, Ali NA, Malik EM. Do Frontline Healthcare Providers Know Enough about Artemisinin-based Combination Therapy to Rationally Treat Malaria? A Cross-Sectional Survey in Gezira State, Sudan. Malar J. 2015; 14(1): 1-7.
9. Toklu H. Promoting Evidence-Based Practice in Pharmacies. Integr Pharm Res Pract. 2015; 4: 127-131.

10. Asibong U, Etokidem A, Akpan U, Archibong F. Malaria: Knowledge, Attitudes and Acceptances of Rapid Diagnostic Test amongst Primary Healthcare Workers in some Selected Local Government Areas, Lagos State. Public Heal Res. 2019; 9(1): 13-21.

11. Ndibuagu EO. Use of Malaria Rapid Diagnostic Tests among Medical Doctors in a Tertiary Hospital, South East Nigeria. Health. 2018; 10(07): 971-984.

12. Nag S, Ursing J, Rodrigues A, Crespo M, Krogsgaard C, Lund O, et al. Proof of Concept: Used Malaria Rapid Diagnostic Tests Applied for Parallel Sequencing for Surveillance of Molecular Markers of Antimalarial Resistance in Bissau, Guinea-Bissau during 2014-2017. Malar J. 2019; 18(252): 1-13.

13. Elmannan AAA, Elmardi KA, Idris YA, Spector JM, Ali NA, Malik EM. Antimalarial Prescribing Practices in Sudan Eight Years after Introduction of Artemisinin-based Combination Therapies and Implications for Development of Drug Resistance. BMC Pharmacol Toxicol. 2015; 16(1). 